\title{
ABSCHLIESSENDER BERICHT NACH DEN DISKUSSIONEN ÜBER DIE VERDUNSTUNG
}

1 Die Verdunstung wird durch 2 Faktoren begrenzt:

a Die Wassermenge, die verdunstet wird und

b Die Energie für die Verdunstung des Wassers.

2 Die extremen Bedingungen von grosser Energiezufuhr und geringem Wasservorrat erschweren die genaue physikalische Untersuchung sehr, insbesonders die Frage der Wasserbewegung in ungesättigtem Boden und Fragen der Pflanzen-Biologie (Feuchtigkeitsspannung). Die landwirtschaftlichen Probleme in den Wüstenzonen sind durch diese Bedingungen charakterisiert.

3 Beim anderen Extrem : "Wasserüberschuss" ist es möglich, bekannte physikalische Gesetze mit Erfolg anzuwenden, die zu dem allgemeinen Grundsatz führen : $\mathrm{Zu}$ einer bestimmten Zeit und an einem bestimmten Ort besteht eine obere Grenze für die Verdunstung von einer gewissen Erntefläche; diese "maximale Verdunstung" kann durch Hinzufügung von Wasser auf die Fläche nicht erhöht werden.

4 Die Physik der Verdunstung (Wasserüberschuss) ist ein Komplex von Energieübertragung und Energieumformung, wobei einige der Energieïbertragungen von aerodynamischen Faktoren gesteuert werden.

5 Die Analyse dieses Komplexes zeigt, dass sowohl die Energieübertragung als auch die aerodynamische Umwandlung im wesentlichen meteorologischen Charakter haben, was zu einem weiteren Grundsatz führt: Die maximale Verdunstung (wie sie im $\$ 3$ definiert wird) wird in erster Linie durch das Wetter bestimmt.

6 Mit gewissen Einschränkungen erscheint die maximale Verdunstung weitgehend unabhängig von der Art des Pflanzenbestandes und der Art des Bodens, auf welchem dieser wächst. Unter diesen Bedingungen erscheint es ratsam, der maximalen Verdunstung einen speziellen Namen zu geben, nämlich: "potentielle Transpiration" (oder "potentielle Evatranspiration"), welche wie folgt definiert wird:

Die potentielle Transpiration ist die Verdunstung von einer grösseren Oberfläche von niederem, grünem Pflanzenwuchs ${ }^{1}$ ), der in vollem Wachstum ${ }^{2}$ ) begriffen ist, den Boden vollkommen beschattet, von gleichmässiger Höhe ist und Wasserüberschuss besitzt.

7 Dieser Begriff einer potentiellen Transpiration ist sogar bei der qualitativen Abschätzung der Verdunstung nützlich.

8 Es gibt verschiedene Verfahren, um quantitative Werte der potentiellen Transpiration zu erhalten. Alle Verfahren machen Gebrauch von meteorologischen Daten, wobei diejenigen Verfahren am besten erscheinen, die sowohl energetische wie aerodynamische Aspekte des Verdunstungsprozesses berücksichtigen.

1) Bei dem gegenwärtigen Zustand der Untersuchungen und der Forschung ist es unmöglich, eine genaue Definition des Ausdruckes "niedriger, grüner Pflanzenwuchs" zu geben. Das Wort "niedrig" betrifft Pflanzenbestände sowohl im Ackerbau wie auch in der Gartenkultur.

2) "Volles Wachstum" bedeutet, dass der Pflanzenbestand in letzter Zeit nicht in seinem Wachstum gestört wurde, wie z.B. : keine Frostschäden oder kein Abmähen. 
9 Physikalische Unsicherheiten in der Abschätzung der potentiellen Transpiration entstehen aus 2 Gründen :

a Unvollständige Kenntnis der Physik von

1 Strahlungsumsatz in freier Luft und unter Glasbedachung (Treibhäuser);

2 Turbulente Übertragungsprozesse der Atmosphäre.

b Die Schwierigkeit der Messung der entsprechenden physikalischen Grössen.

10 Biologische Unsicherheiten entstehen aus vielen Gründen : z.B. die Morphologie der einzelnen Pflanzenarten, der physiologische Zustand und die Aktivität der Pflanze. Obwohl einige dieser Probleme offensichtlich physikalischen Charakter haben, ist es klar, dass die Zusammenarbeit mit den Biologen wesentlich ist bei dem Versuch, die vorhandenen Schwierigkeiten zu beseitigen.

11 Trotz der Nachteile, die im $\S 9$ u. 10 angeführt sind, ist es klar, dass die Verdunstung in der Landwirtschaft (bei Wasserüberschuss) im Grunde eine physikalische Erscheinung ist. Für viele praktische Zwecke kann die Verdunstung durch meteorologische Faktoren beeinflusst und quantitativ ausgedrückt werden, wenn die entsprechende Werte der meteorologischen Faktoren bekannt sind.

12 Mit Präzisionsinstrumenten, einer sehr geschickten Messtechnik und etwas komplizierten Berechnungen ist es möglich, die wirkliche Verdunstung abzuschätzen. Diese Arbeit ist von fundamentaler Bedeutung in der physikalischen Meteorologie. Die Ergebnisse werden sicher für die Agrarwissenschaften und schliesslich auch für die Agrarpraxis von Nutzen sein.

13 Gegenwärtig entstehen jedoch Schwierigkeiten, wenn die Ergebnisse, die über kleine Zeitintervalle gewonnen wurden, auf Zeiträume angewendet werden, die in der Landwirtschaft oder in der Hydrologie üblich sind. Vorerst müssen jedoch für die Praxis diese unvollkommenen Erkenntnisse genügen. Es müssen dazu Methoden verwendet werden, die leichter durchführbare Messungen verwenden und einfachere Berechnungsverfahren. Es existieren zahlreiche praktische Probleme, in denen eine Näherungs-Methode vertretbar ist.

14 Derartige Methoden sind vorhanden, aber nicht einfach zu prüfen wegen der Schwierigkeit, eine genaue Wasserbilanz unter Freilandbedingungen anzugeben. Es bestehen jedoch genügend Freiland-Beobachtungen, die bestätigen, dass die Verdunstungsschätzungen, die auf diese Art erreicht wurden, für Probleme der Bewässerungsentwicklung und Bewässerungspraxis anwendbar sind, besonders wenn die Bewässerung so stark ist, dass sie einen Wasserüberschuss ergibt. Die Möglichkeiten einer Schätzung der potentiellen Transpiration sind abhängig von der Anzahl der meteorologischen Beobachtungen; die Genauigkeit dieser Schätzung ist begrenzt, wie im $\S 9$ u. 10 angegeben wurde. Es erscheint gegenwärtig riskant, mit den Näherungs-Formeln den Wasserverlust über kürzere Zeiträume abzuschätzen.

15 Die Berechnung der Verdunstung von grossen, freien Wasserflächen erscheint genügend genau $\mathrm{zu}$ sein.

16 Die Anwendung der Wasserbilanz in Gegenden mit ungleichen Regenfällen verlangt die Abschätzung der Differenz zwischen potentiellem und wirklichem Wasserverlust. Die Resultate enthalten eine beträchtliche Unsicherheit selbst in feuchten Gegenden, wo jedoch trotzdem nützliche Resultate gefunden wurden.

17 Diese Schätzungen sind notwendig, weil die Kenntnis des Wassertrans- 
portes durch den Boden und durch die Pflanzen ungenügend ist. Sie wird ungenügend bleiben, bis weitere biologische und physikalische Forschungen durchgeführt werden. Die gegenwärtigen Messverfahren für den Wassergehalt des Bodens genügen nicht für diese Forschungen; dieser Mangel begrenzt die Möglichkeiten für einen raschen Fortschritt; verbesserte Methoden sind dringend erforderlich.

18 Es ist wesentlich, dass die Forschungsarbeiten der Physiker, Hydrologen, Biologen, Landwirten und Kulturtechnikern in enger Zusammenarbeit erfolgen. Eine derartige Zusammenarbeit sollte international sein und in allen Stadien der Forschung durchgeführt werden. Z.B. ist es in instrumenteller Hinsicht sehr wünschenswert, dass die Wissenschaftler in verschiedenen Ländern, sowohl ihre Erfahrungen über Misserfolge als auch über Erfolge austauschen. Es ist nicht immer ausreichend, wenn man sich auf die Veröffentlichungen verlässt. Es sollten die Möglichkeiten für persönliche Aussprachen sowohl vor als auch während der Untersuchungen vorhanden sein. Hierdurch könnte viel Zeit und Geld erspart werden, da besonders kritische Bemerkungen nicht durch die Auswertung der Literatur gefunden werden.

19 Abschliessend möchten wir folgende 2 Ansichten bestätigen :

a Obwohl die Wichtigkeit des Wassers für die Menschheit überall anerkannt wird, entsprechen die Forschungsarbeiten für die Kontrolle des Wasserhaushalts in keiner Weise dieser Wichtigkeit.

b Die Entdeckung und Formulierung der Gesetze der Wasserbewegung muss den Physikern arbeitend auf diesem Gebiet überlassen werden.

20 Forschung und Anwendung der Forschungsergebnisse sollen gleichzeitig erfolgen, da es weder notwendig noch wünschenswert ist, zu warten, bis die Wissenschaftler vollkommene Resultate erzielen, bevor sie praktischen Zwecken dienen können. Die doppelte Aufgabe für den Physiker ist klar : Seine cigenen Kenntnisse zu erweitern und seine Hilfe zu verstärken, überall wo sie gebraucht wird. 